ARTÍCULO: CONCENTRACIÓN URBANA EN AMÉRICA LATINA. RELACIONES ENTRE MACROCEFALIA Y DESCENTRALIZACIÓN FISCAL.

\title{
CONCENTRACIÓN URBANA EN AMÉRICA LATINA. RELACIONES ENTRE MACROCEFALIA Y DESCENTRALIZACIÓN FISCAL.
}

\author{
Urban concentration in Latin America. relationships between microcephaly and fiscal decentralization \\ Concentração urbana na América Latina: relações entre microcefalia e descentralização fiscal
}

\section{RESUMEN}

El documento presenta un repaso de los principales modelos teóricos de la descentralización fiscal y su efecto en la composición demográfica de las regiones de cada país, contrastándola con las teorías de la primacía urbana y la macrocefalia, haciendo especial énfasis en su incidencia en América Latina. Mediante el análisis de datos y la revisión bibliográfica se constató que la relación entre gasto público, tributación subnacional y reducción de la primacía urbana, solo fue estadísticamente significativa en países por fuera del contexto latinoamericano, lo cual refuerza el llamado a profundizar en la comprensión de este fenómeno y la construcción de modelos teóricos y analíticos que conlleven a un gasto público descentralizado más eficiente que coadyuve a la reducción de la primacía urbana en América Latina.

PALABRAS CLAVE: macrocefalia, descentralización, primacía urbana, demografía, federalismo fiscal.

\section{Manuel Alberto Gómez González ${ }^{1}$}

mangom08@ucm.es

ORCID:http://orcid.org/0000-0003-4770-7989

1 Universidad Complutense de Madrid, Espanha

Sometido 13-11-2019 Aprobado 23-04-2020

Evaluado por el sistema double blind review.

DOI: http://dx.doi.org/10.12660/cgpc.v25n82.80599 


\section{ABSTRACT}

The document presents an account of the main theoretical models of fiscal decentralization and its impact on the demographic composition of the regions in each country, contrasting it with urban primacy and macrocephaly theories, with a special emphasis on their incidence in Latin America. Through data analysis and literature review, it was found that the incidence of public spending and subnational taxation on the reduction of urban primacy was only statistically significant in countries outside the Latin American context, which reinforces the call to deepen the understanding of this phenomenon and the construction of theoretical and analytical models that lead to a more efficient decentralized public expenditure that contributes to the reduction of urban primacy in Latin America.

KEYWORDS: macrocephaly, decentralization, urban primacy, demography, fiscal federalism.

\section{RESUMO}

O trabalho apresenta um relato dos principais modelos teóricos da descentralização fiscal e seu impacto na composição demográfica das regiões de cada país, contrastando-o com as teorias de primazia urbana e mácrocefalia, com ênfase especial em sua incidência na América Latina. Por meio da análise dos dados e revisão da literatura, verificou-se que a incidência de gastos públicos e tributação subnacional na redução da primazia urbana foi estatisticamente significativa apenas em países fora do contexto latino-americano, o que reforça a necessidade de aprofundar a compreensão desse fenômeno e a construção. de modelos teóricos e analíticos que levam a um gasto publico descentralizado mais eficiente qué contribui para a redução do primado urbano na America Latina.

PALAVRAS-CHAVE: macrocefalia, descentralização, primazia urbana, demografia, federalismo fiscal.

\section{INTRODUCCIÓN}

En el marco del debate de la descentralización y el federalismo fiscal, la primera generación teórica denominada "normativa", reflejada en los trabajos de Musgrave (1984), Oates (1974) y Tiebout (1959), hace énfasis en los beneficios de la autonomía territorial, en tanto aumenta la eficiencia del gasto mediante la reducción de las asimetrías de información entre oferta y demanda y permite acercar a niveles óptimos la utilidad marginal del gasto público y el costo marginal de la tributación, pues la movilidad de los contribuyentes para ubicarse en la jurisdicción que más se ajuste a sus preferencias, denominada selección con los pies, y la cercanía con los burócratas, facilita conocer más fácilmente las preferencias locales y así financiar más oportunamente las demandas de la ciudadanía. Este esquema presupone que los gobiernos regionales cuentan con capacidades de provisión de bienes públicos diferenciados y con una hacienda públi- ca subnacional altamente autónoma.

Sin embargo, los dos principios básicos del modelo (autonomía territorial y haciendas públicas locales autosuficientes) no son una realidad, la mayoría de los territorios no tienen la capacidad fiscal de proveer bienes y servicios públicos de manera autónoma y requieren la financiación (o subvención) de territorios con mayores posibilidades de recaudación. Así, surgió una segunda generación teórica de la descentralización que, atenuando a los postulados de autonomía y eficiencia de la primera y poniendo énfasis en la equidad como elemento definidor de las instituciones descentralizadoras, buscó impulsar reformas encaminadas a establecer mecanismos de financiación conducentes a reducir las inequidades horizontales mediante sistemas de trasferencias (Garzarelli, 2004; Oates, 2005; Weingast, 2007).

El problema de esta segunda generación es que las trasferencias tienden a generar los 
problemas de riesgo moral y asimetrías de información entre el principal y el agente, descritos el modelo de maximización de presupuesto propuesto por Niskanen (1974). La mayoría de los diseños institucionales con sistemas de trasferencias no condicionadas o "block grants", conllevaron a problemas de gasto ineficiente, aumento del gasto público y corrupción. La solución implementada fue el condicionamiento de las trasferencias "matched grants", conllevando a un direccionamiento del gasto por parte del principal (generalmente el gobierno central), reduciendo la autonomía de los agentes (gobiernos locales), buscando disminuir las brechas regionales y garantizar la provisión de bienes públicos, sacrificando la autonomía, elemento central de la descentralización.

Así, encontramos una tensión teórica (reflejada en la realidad) en donde, por un lado, se propende por la eficiencia y la autonomía local, pero por el otro se introducen mecanismos de trasferencias que pretenden reducir las brechas interregionales de cada país, las cuales a su vez distorsionan las decisiones y la autonomía local o conllevan a riesgos asociados al Modelo de maximización de presupuesto, además de restar autonomía local.

Los modelos teóricos y sus consiguientes diseños institucionales tuvieron origen en Estados Unidos y fueron mutando según el país. España y Canadá desarrollaron sistemas de descentralización que buscaron el fortalecimiento progresivo de la autonomía local, Alemania apostó a la construcción de una descentralización cooperativista con alta participación de las regiones en las decisiones federales, Francia diseño su sistema basado en un modelo centralista, en donde la desconcentración geográfica y la delegación han primado. Por su parte Latinoamérica ha avanzado en la implementación de modelos mixtos, inspirados esencialmente por Estados Unidos y Francia.

Además de los efectos en la eficiencia del gasto público y la reducción de las brechas horizontales entre las regiones de cada país, los modelos teóricos indican que la descentralización fiscal tiene incidencia en la configuración demográfica de un país. En esa línea, Tsai (1990) analizó el efecto del gasto público (de las políticas de industrialización) en la concentración demográfica de Taiwán; Boisier (1992) estudia el efecto de la descentralización en la reducción de la macrocefalia y la trasformación productiva en América Latina; en 2017 el Comité Europeo de las Regiones (2017) señaló en los puntos 22 y 23 de su informe sobre las migraciones del mediterráneo, la necesidad de fortalecer la provisión de servicios públicos locales y mejorar la calidad de vida de la población para reducir los incentivos a migrar; la OCDE (2020) en su análisis sobre descentralización en Portugal señala la importancia del gasto público en la consolidación de un mercado laboral descentralizado por fuera de la capital.

La bibliografía y los datos son consistentes al indicar cómo las particularidades de América Latina en su proceso de urbanización, migraciones internas e historia demográfica, la hacen una región con necesidad de desarrollos académicos que permitan su comprensión. Entre 1950 y 2020 América Latina paso de tener 7 ciudades con más de un millón de habitantes, a tener 71 , siendo superada solo por Asia como la región con mayor crecimiento, de las 15 ciudades más 


\section{Manuel Alberto Gómez González}

pobladas del mundo, 5 son latinoamericanas y concentran una población que representa entre el 17 y el $33 \%$ de la población total del país respectivo. La dependencia de la periferia al centro, el crecimiento de sus aglomeraciones urbanas y el alto nivel de crecimiento demográfico en las ciudades de mayor tamaño son particularidades de América Latina (Arango, 2003; Cortés, 2017; Cuervo, 2004).

Tomando de la medicina el concepto de macrocefalia, Castells (1973) acuño la idea para describir los procesos de urbanización en América Latina, explicando el crecimiento exponencial de las ciudades capitales de la región y sus efectos en la calidad de vida, la gobernabilidad del territorio y la desigualdad. Este proceso de concentración urbana transformó las formas como las administraciones públicas locales y nacionales de la región se organizaron para gobernar todas sus regiones y proveer bienes y servicios a la población.

Países como Estados Unidos, Alemania, Francia, España o Canadá han construido sus modelos de relaciones intergubernamentales basados en las dos generaciones teóricas, sin embargo, las características de América Latina no se coligen completamente con las teorías de la descentralización. Las características económicas, demográficas culturales y fiscales de la región requieren la construcción de nuevos desarrollos teóricos capaces de integrar las variables demográficas y migratorias, con el fin de aportar a la reforma de los sistemas de relaciones intergubernamentales en los países latinos. El documento presenta el resumen de un modelo estadístico que compara las posibles correlaciones e incidencias entre la macrocefalia urbana en América Latina, el gasto público y la recaudación subnacional, buscando aportar a elementos para un nuevo modelo teórico de la descentralización fiscal para la región.

En el primer apartado, se hace un recuento de los modelos teóricos del federalismo fiscal, su incidencia en el gasto público descentralizado y la participación de los gobiernos subnacionales en los tributos de cada estado, seguidamente se presenta la evolución de la primacía urbana y la macrocefalia en América Latina. El tercer apartado expone la metodología usada para la construcción de la base de datos y resume los resultados del análisis de la relación entre el gasto y recaudación pública descentralizada y la primacía urbana de los países de la muestra, así como las particularidades de los países de América Latina. Finalmente se exponen algunas consideraciones finales.

\section{EL FUNDAMENTO TEÓRICO DE LA DES- CENTRALIZACIÓN}

La propuesta teórica de la descentralización tiene sus orígenes en la crisis económica de la posguerra a mediados de la década de los 60's, pues conllevó a los países a replantear sus diseños institucionales en torno a cómo, el Estado y el mercado, venían proveyendo bienes y servicios. Particularmente para países en vías de desarrollo se comenzaron a gestar movimientos académicos y políticos que abogaron por un cambio en el modelo conocido como modelo de sustitución de importaciones; el principal razonamiento fue el crecimiento desbordado del aparato burocrático, el agotamiento del estado de bienestar y una ineficiencia e inflexibilidad del gasto público, consumidor buena parte de los re- 
cursos producidos por el sector privado, así como una fuerte restricción a la conformación de mercados abiertos y competitivos (Díaz Flores, 2002; Robert \& Harper, 2004). En este contexto, en los Estados Unidos, Tiebout (1959) planteó la necesidad de repensar la provisión de bienes públicos, cuestionando la forma tradicional como se definía a quiénes y cómo se proveían dichos bienes; este nuevo planteamiento introdujo criterios de eficiencia, buscando reducción de las asimetrías de información en la toma de decisiones públicas. Los procesos de descentralización se entienden entonces como un proceso de traspaso (o devolución) de competencias, recursos y capacidades del gobierno central hacia los gobiernos subnacionales (Díaz Flores, 2002; Finot, 2001).

Desde la economía institucional, la descentralización ha buscado el aumento de la eficiencia del estado en la ejecución de sus políticas públicas, así como una reducción de las desigualdades horizontales o regionales. Estos dos objetivos han fundamentado dos generaciones teóricas que han marcado y orientado las reformas institucionales de la mayoría de países con diseños institucionales verdaderamente descentralizados (Cibils \& Ter-minassian, 2016,).

Finanzas Públicas, Bienes Públicos y Maximización del Bienestar Social

Para entender el "teorema de la descentralización" propuesto por Oates, es necesario ubicarse en una concepción keynesiana de la economía institucional, en donde se asume que el sector público tiene la capacidad y la responsabilidad de diseñar instituciones capaces de regular el mercado para corregir sus fallos y garantizar el acceso a ciertos bienes y servicios públicos. En esta línea, la primera generación del federalismo fiscal, y consecuentemente de la descentralización, tiene parte de su origen en las propuestas hechas por Samuelson, Arrow y Musgrave (Oates, 2005; Qian \& Weingast, 1997).

A partir de lo propuesto por Samuelson (1955), la primera generación de teorías de la descentralización toma como partida los planteamientos fundados en la economía del bienestar, la asignación correcta de roles entre el mercado y el estado, y retoma el teorema de Arrow (1970), indicando la imposibilidad de construir modelos de decisión que logren reunir las preferencias racionales de toda la población cuando existen más de dos alternativas, evidenciando la inexistencia de una función de utilidad social plena. En otras palabras, pone el énfasis en la multiplicidad de preferencias presentes en cada un país y cómo esas diferencias no pueden ser atendidas eficientemente por una oferta pública uniforme o centralizada.

Musgrave (1984) planteó, la necesidad de introducir nuevos elementos de análisis al estudio de la economía del sector público, señalando como, además de tener funciones relacionadas con la asignación eficiente de recursos en una economía, un estado tiene entre sus funciones la de estabilizar la economía y buscar una redistribución de la renta, propendiendo por la reducción de las inequidades verticales y horizontales.

La Primera Generación Teórica del Federalismo Fiscal, el Modelo de Tiebout.

Oates no toma en consideración como fundamentales los aportes del modelo de Tiebout (1959) sin embargo, es innegable como 


\section{Manuel Alberto Gómez González}

en su teoría del gasto público local y su federalismo fiscal, Tiebout abrió la puerta para que la economía institucional comenzara a buscar caminos hacia la eficiencia en la provisión de bienes públicos a través de los gobiernos subnacionales.

El modelo contiene dos elementos, por un lado, esquemas de provisión de bienes públicos diferenciados según cada localidad, donde los habitantes de cada lugar podrán elegir la cantidad y características de los bienes a recibir, conllevando a una hacienda pública subnacional específica en las diferentes jurisdicciones, permitiendo fijar niveles de tributación, que se correspondan con la provisión de los bienes decididos por los consumidores locales. En suma, la teoría señalo cómo las diferentes jurisdicciones compiten por los contribuyentes permitiéndoles elegir en cuál de ellas asentarse de acuerdo con sus preferencias, relacionadas con la característica de los bienes públicos y cuánto están dispuestos a pagar por ello a través de su respectiva carga tributaria, esto es lo que se conoce como, la elección con los pies. (Tiebout, 1959)

La teoría del federalismo fiscal asume que, si se proveen bienes públicos diferenciados, elegidos por los contribuyentes, la oferta responde más eficientemente a las preferencias y necesidades de la demanda, reduciendo las asimetrías de información, pues la elección con los pies y la cercanía con los burócratas facilita el conocimiento de la realidad y predilecciones locales.

"Las personas en cuyo esquema de preferencias los beneficios que ofrece un elevado nivel de servicios públicos son superiores a los costes que implica un ni- vel alto de presión fiscal se desplazarán a las jurisdicciones de mayor gasto público. Y lo contrario harán quienes prefieran un nivel más bajo de presión fiscal a cambio de un menor gasto público. De esta forma las personas y las empresas "votaran con los pies" buscando la combinación gasto público/presión fiscal que mejor se adapte a sus preferencias." (Albert, Biazzi, \& Cabrillo, 2011, p. 18)

Del Federalismo Fiscal al Teorema de la Descentralización de Oates

Para Oates (2005) el principal problema de la primera generación teórica de la descentralización está asociado a la movilidad de la demanda. En la propuesta hecha por Tiebout, los electores tendrían la posibilidad de una plena libertad para elegir la localidad para residir. Frente a este argumento hay dos críticas, la primera, en la realidad, los factores movilidad de una localidad a otra están asociados a muchas condiciones diferentes a los diseños tributarios diferenciados: factores laborales, culturales, idiomáticos, familiares, geográficos entre muchos otros son variables que condicionan la movilidad de las personas entre una región y otra, con lo cual, de entrada se desestima la idea de los incentivos para la migración entre regiones por motivos meramente tributarios (Oates, 2005).

Un segundo argumento está asociado a la necesidad de la movilidad interregional como condición para concebir modelos descentralizados. Para Oates, en ello radica la gran critica al modelo de Tiebout pues, aun en ausencia total de movilidad, existe una demanda de bienes públicos ya asentada en cada territorio, la cual tiene diferentes preferencias 
CONCENTRACIÓN URBANA EN AMÉRICA LATINA. RELACIONES ENTRE MACROCEFALIA Y DESCENTRALIZACIÓN FISCAL.

y por lo tanto exige una respuesta diferencia para aumentar la eficiencia del gasto público (Garzarelli, 2004; Oates, 2005).

Si el estado tiene funciones y responsabilidades a la hora de proveer ciertos bienes públicos, y esa provisión no puede darse de manera uniforme en todas las localidades de cada país, dada la imposibilidad de construir ofertas públicas ajustadas a las preferencias cambiantes y diferenciadas de cada localidad, el teorema de la descentralización plantea que, con igual cantidad de recursos, un gobierno local puede proveer un bien 0 servicio público más ajustado a las necesidades y o referencias de la demanda, con lo cual la utilidad social será mayor y consecuentemente, será más Pareto - eficiente la intervención del estado. (Arrow, 1970; Oates, 1974; Samuelson, 1955)

Pero además de ello, las funciones económicas del sector público sugeridas por Musgrave dieron luces a la construcción de un marco teórico que dilucida la diferenciación de responsabilidades y competencias de los gobiernos territoriales y el gobierno nacional. por un lado, las funciones asociadas con la estabilidad macroeconómica se le asignan al gobierno nacional pues la emisión de la moneda, el control de la deuda, la fijación de tasas de interés y demás acciones, solo pueden ser adelantadas, en principio, por gobiernos con una capacidad de intervención fuerte, cosa que en la mayoría de los gobiernos locales no sucede.

En cuanto a la función de redistribución de la renta o de reducción de brechas horizontales y verticales, se entiende que buena parte de esta tarea debe dejársele a los gobiernos centrales en tanto tienen la capacidad de re- caudar tributos en las diferentes localidades (unas con mayor capacidad fiscal que otras) y distribuir entre esas mismas localidades para garantizar un acceso mínimo a ciertos bienes y servicios cuyo consumo produce externalidades positivas que el estado procura maximizar mediante la internalización los costos de su provisión ( Musgrave, 1984; Oates, 1999, 2005).

Estos desarrollos hicieron parte de la fundamentación teórica que ayudó al consenso de Washington a ofrecer una receta para replantear el Modelo de Estado vigente hasta la década de los 80`s. Según Furlan (2012), además de la reducción del aparato burocrático, la limitación de la intervención del Estado en el mercado, la apertura económica, la reducción de las barreras de los mercados locales y la tercerización en la prestación de servicios, la descentralización fue una de las aplicaciones prácticas propuestas por el consenso para modificar los diseños institucionales de la fecha.

La mayoría de países en vías de desarrollo iniciaron a mediados de los 80's, reformas encaminadas a iniciar una transferencia 0 devolución de poder desde el gobierno nacional a los territoriales, "en parte en respuesta a presiones políticas en favor de la autonomía local, pero también de una mayor eficiencia en la prestación de los servicios públicos" (Kahkonen, Satu, Lanyi, 2001, p1). Como parte del apoyo teórico a esta serie de reformas institucionales, el teorema de la descentralización de Oates, así como desarrollos posteriores, pusieron énfasis en la aplicación práctica del federalismo fiscal, buscando clarificar cuáles son los elementos centrales a tener en cuenta a la hora de diseñar instituciones descentralizadoras, 


\section{Manuel Alberto Gómez González}

como mecanismo de redistribución del poder del Estado (Oates a. E., 1999).

«Los trabajos de Oates y Buchanan, publicados en los años 70, (...) llevaron en los años ' 80 y ' 90 del siglo pasado a justificar teóricamente y celebrar políticamente las ventajas de la descentralización o la devolución de poderes, posición adoptada por el Banco Mundial y el Fondo Monetario Internacional en sus recetas a los países subdesarrollados y a los "en vías de desarrollo", así como a los países exsocialistas para facilitar un crecimiento económico rápido. Y desde la ciencia política, se consideró que la división de la soberanía entre diferentes niveles de gobierno aseguraba el camino de la paz y la estabilidad en sociedades divididas por fuertes diferencias lingüísticas o étnicas"» (Braña, 2006, p. 69).

En resumen, los desarrollos teóricos de la descentralización se pueden categorizar en dos grandes generaciones, la primera denominada normativa, se fundamentó en los trabajos de Musgrave (1969), Oates (1972) y Tiebout (1961) y "enfatizó los beneficios de la descentralización en términos de eficiencia en la asignación de recursos. La teoría se apoyaba en supuestos acerca de la facilidad con que la descentralización podría dar lugar a una gobernanza eficaz:" (Cibils \& Ter-minassian, 2016, p.2).

Una segunda generación de literatura especializada denominada como positiva, hizo especial énfasis en la necesidad de aterrizar los postulados de eficiencia del federalismo fiscal a las condiciones de países en vías de desarrollo en donde, como se explicó hasta el momento, no es posible identificar con claridad los supuestos necesarios para aprovechar la eficiencia indicada por la descentralización. (Cibils \& Ter-minassian, 2016).

\section{PRIMACÍA URBANA Y MACROCEFALIA EN AMÉRICA LATINA}

Además de cómo distribuir competencias y recursos en el territorio de un país para garantizar la mayor eficiencia en el gasto público y una reducción de las brechas horizontales, una de las cuestiones fundamentales de las relaciones intergubernamentales y el análisis territorial, es el tamaño ideal de una localidad, si es que existe, y cuáles reglas lo determinan. La primacía urbana es la relación de dominio y aumento de la importancia relativa de la ciudad principal de determinado país, en relación con las demás urbes. Si bien es un fenómeno asociado con la concentración demográfica urbana, va más allá, pues se centra en analizar el peso relativo y las relaciones de las ciudades de un sistema urbano. El cambio en las relaciones entre ciudades se ve reflejado en una profundización de la relevancia de la ciudad principal al concentrar la población, actividad económica y generación de riqueza, consolidándola como el centro de poder y toma de decisiones políticas (Cuervo, 2004).

La primacía urbana parte de la concepción de una ciudad dominante a partir de la cual se configuran las redes de trasporte, referentes culturales, dinámicas de poder y tamaño de las ciudades subordinadas a la principal. Es una variación de las ideas de centro periferia. Cuervo (2004) propone la reflexión en torno a la primacía urbana, más allá de un mero análisis de concentración demográfica y desmarca la discusión de una cuestión meramente normativa sobre el tamaño ideal 
de un centro urbano. En ese marco, el autor señala la importancia de ampliar el debate y profundizar en la compresión del fenómeno de la primacía urbana, pues permite entender en contexto, la relación económica, cultural política y espacial de la red de ciudades de cada país, permitiendo un análisis mucho más enriquecedor.

Por su parte, la macrocefalia es un concepto tomado de la medicina que indica una relación patológica de un sistema respecto de su principal órgano. Es una desproporción de la primacía urbana de la ciudad capital. La concentración desproporcionada de población, actividad económica, cultural y poder político puede llevar a una relación desequilibrada entre la principal y las demás ciudades, centralizando la generación de riqueza y teniendo un efecto nocivo en la profundización de las inequidades horizontales (Castells, 1973; Cuervo, 2004).

Para entender adecuadamente el proceso de consolidación de la primacía urbana, y su posible macrocefalia, se debe partir del contexto histórico y cultural en el caso de América Latina, la literatura muestra cómo este proceso ha sido influido por su modelo de desarrollo y de industrialización (Montoya, 2009).

Cuervo (2004) señala que la influencia colonial sobre la primacía urbana no está realmente marcada, salvo los casos de Lima y la Nueva Granada, el surgimiento de las ciudades principales de América Latina está más asociado a fenómenos post coloniales en donde el principal factor fue el proceso de industrialización. Según el autor, en un punto cero, indicado el período entre 1860 y 1980, el índice de población total del país que habita en las ciudades principales (IPT) comparado entre América Latina y Europa fue muy similar $5,5 \%$ y $4,95 \%$ respectivamente, sobresale con diferencia el índice para Centroamérica en donde el porcentaje promedio de población concentrada en la ciudad principal fue del 9,5\%, lo cual el autor lo atribuye al tamaño (de menor escala) de los países centroamericanos, con excepción de México.

Siguiendo los datos aportados por Cuervo (2004) la tasa de variación del índice refleja comportamientos disimiles entre América Latina, especialmente Suramérica y Europa. Para 1970 el IPT máximo en Latinoamérica fue de $23,1 \%$ mientras el de Europa fue de $14,7 \%$. La tasa de variación de la población concentrada en las ciudades principales en el periodo en mención fue de $22 \%$ para Latinoamérica, frente a un $8 \%$ de Europa.

Estos datos evidencian que, en América Latina,

"la fase de capitalismo dependiente de la primera mitad del siglo $\mathrm{XX}$, se experimentó un crecimiento urbano dado por el incremento de la población en las ciudades y por el aumento del número de ellas. (...) Esta tendencia en concentrar el desarrollo urbano en las zonas directamente articuladas al sistema de dependencia, o sea, donde se produjo la expansión y cambio de la economía urbana, estimularía la atracción de población a estas áreas desde otras ciudades y localidades de mayor subdesarrollo. Produciéndose así un desnivel al interior de las sociedades en términos de desarrollo y modernidad". (Cortés, 2017, p. 6). 
Así, Entre 1950 y 2020, según cálculos propios a partir de UN (2015) América Latina pasa de tener 7 ciudades con más de un millón de habitantes, a tener 71 , siendo superada solo por Asia como la región con mayor crecimiento, de las 15 ciudades más pobladas del mundo, 5 son latinoamericanas y concentran una población que representa entre el 17 y el $33 \%$ de la población total del país respectivo.

La dependencia de la periferia al centro y el marcado crecimiento demográfico en las ciudades de mayor tamaño son particularidades sobresalientes en América Latina. La primacía urbana refleja la progresiva concentración de poder político y económico de la ciudad principal de cada país, que se expresa especialmente en un aumento de la proporción de su población, lo cual tendrá efectos en la concertación del gasto público y la recaudación tributaria, en tanto el gobierno nacional, asentado en las capitales, controla la ejecución y la recaudación. (Clemens \& Veldhuis, 2013)

Al analizar conjuntamente los postulados teóricos y la evolución de los datos, se colige una posible relación entre gasto público y primacía urbana, en tanto todos los modelos indican la incidencia del uno en el otro. Bien sea porque la movilidad por incentivos tributarios ajusta la oferta y demanda pública, o bien porque el gasto público local puede reducir las brechas interregionales y con ello desincentivar la concentración demográfica en las ciudades principales. La relación entre primacía y gasto descentralizado es un elemento a considerará en el análisis, con lo cual se avanzará en la discusión en torno a la eficiencia del gasto público y el tamaño de las ciudades.

\section{INCIDENCIA DEL GASTO PÚBLICO DES- CENTRALIZADO EN LA MACROCEFALIA EN AMÉRICA LATINA: PRESENTACIÓN DEL MODELO}

Hasta ahora, se han presentado los modelos teóricos de la descentralización, la macrocefalia y la primacía urbana, haciendo especial énfasis en las particularidades de América Latina a la hora de aplicar dichos modelos.

Las dos generaciones de la descentralización sugieren la existencia de una relación entre concentración demográfica y descentralización fiscal. Una de las principales críticas de Oates (2005) al modelo normativista de federalismo fiscal de Tiebout es asumir una libre movilidad de los ciudadanos, basada únicamente en incentivos tributarios y de gasto público. para la segunda generación ya existe una demanda local asentada que debe ser atendida según criterios descentralizadores, lo cual tendrá efectos en la calidad de vida y en los incentivos a la hora de migrar.

Así mismo, la literatura muestra cómo América Latina tiene unas condiciones demográficas particulares en la relación entre las capitales y las demás localidades de los países de la región, pues están marcadas por una alta dependencia y primacía urbana, llegando a insinuar una macrocefalia urbana, dada la gran concentración demográfica y la relevancia económica relativa de las capitales frente a las demás ciudades.

Basados en los postulados teóricos y en las condiciones particulares de dependencia urbana de América Latina, este apartado presenta un análisis cuantitativo que pretende aportar a la discusión en torno a la influencia 
CONCENTRACIÓN URBANA EN AMÉRICA LATINA. RELACIONES ENTRE MACROCEFALIA Y DESCENTRALIZACIÓN FISCAL.

de la descentralización fiscal en la primacía urbana de América Latina, dilucidando algunas particularidades que señalan la necesidad de la construcción de nuevos modelos teóricos explicativos de las particularidades de la región.

Para el análisis se usó una muestra de 38 países, 16 de Latinoamérica (Argentina, Bolivia, Brasil, Chile, Colombia, Costa Rica, República Dominicana, Ecuador, El Salvador, Guatemala, Honduras, México, Panamá, Paraguay, Perú, Uruguay) y 22 de otras regiones (Australia, Canadá, China, Croacia, Francia, Alemania, India, Irlanda, Israel, Italia, Holanda, Nicaragua, Noruega, Polonia, Portugal, Puerto Rico, España, Suiza, Suecia, Turquía, Reino Unido y Estados Unidos).

Se construyó una base de datos no balanceada para todos los países de la muestra, que consigno la información relacionada con población, gasto y hacienda pública descentralizada para cada uno de los casos de estudio. Para la información relacionada con población y demografía, se uso la información proporcionada por UN (2015) en donde se cruzó la población total con la población de las ciudades capitales, (incluyendo las zonas conurbadas de cada una), para calcular el índice de población total IPT, entendido como la proporción del total de la población del país que habita en las ciudades principales (Cuervo, 2004).

En cuanto a la información relacionada con gasto público y hacienda pública descentralizada se usaron las bases de datos proporcionadas por OCDE (2016) e IFM (2018). EI indicador de gasto público descentralizado, está definido como la proporción del gasto público total de cada país ejecutado por los gobiernos subnacionales. Por su parte el indicador de hacienda pública descentralizada refleja la proporción de la recaudación tributaria y no tributaria total de cada país, percibida por los gobiernos subnacionales. En los dos casos, los gobiernos subnacionales se entienden como la suma de todos los niveles de gobierno diferentes del central. Los datos disponibles son de 2015.

Para realizar el análisis, se procedió a descartar los datos incompletos, y se cruzo la información fiscal con los indicadores demográficos del 2015, con lo cual se balaceó la base de datos usada.

El modelo introduce tres variables, IPT, gasto público descentralizado y recaudación descentralizada. Con el fin de lograr mayor estabilidad en el modelo y reducir la distorsión de los valores atípicos, se procedió a calcular el logaritmo de las tres variables, permitiendo una observación más completa del comportamiento de estas, así como su interacción.

Al analizar las correlaciones, se encontró que la variable dependiente, el logaritmo de la población de las ciudades capitales (In_ prop_pobl_cap), tiene una relación negativa con el logaritmo del gasto público descentralizado (In_exp_subn) y con el logaritmo de la recaudación descentralizada (In_rev_subn). Sin embargo, existe colinealidad entre las dos variables fiscales, en tanto al aumentar la recaudación aumenta el gasto, y al aumentar el gasto requiere un aumento de la recaudación. Si bien es cierto que esta relación no es determinística, esta situación impide la construcción de un modelo multivariado. Véase la tabla 1. 


\section{Manuel Alberto Gómez González}

Tabla 1. Correlaciones entre población de ciudades capitales, gasto público descentralizado y recaudación descentralizada.

\begin{tabular}{l|l|l|l}
\hline Correlaciones de Pearson & $\begin{array}{l}\text { Ln_prop_population_cap_ } \\
\text { city_total }\end{array}$ & $\begin{array}{l}\text { Ln_rev_prop_ } \\
\text { sub }\end{array}$ & $\begin{array}{l}\text { Ln_exp_ } \\
\text { prop_sub }\end{array}$ \\
\hline $\begin{array}{l}\text { Ln_prop_population_cap_.city_ } \\
\text { total }\end{array}$ & 1 & $-.573^{* *}$ & $-.544^{* *}$ \\
Ln_rev_prop_sub & $-.573^{* *}$ & 1 & $.864^{* *}$ \\
Ln_exp_prop_sub & $-.544^{* *}$ & $.864^{* *}$ & 1 \\
\hline
\end{tabular}

Fuente: elaboración propia a partir de los datos de (IFM, 2018; OCDE, 2016; UN, 2015)

Esta relación negativa entre población de ciudades capital y descentralización fiscal sugiere que, a un aumento del gasto la recaudación descentralizada reduce la concentración demográfica de las ciudades principales. Sin embargo, al analizar en detalle esta relación se encuentran elementos relevantes para ampliar esa conclusión.

En primer lugar, se encontró que al aplicar la prueba de significación y calcular el estadístico, la relación entre población y recaudación, a pesar de tener un índice de correlación significativa para toda la muestra, para los países de América Latina, no hay relación estadística entre las variables, en tanto el estadístico t fue de -1.926 y su grado de significancia fue de 0.090 , sobrepasando los niveles críticos y descartando una posible relación explicativa.

En el caso de los países de las demás regiones, se anula igualmente la conclusión de una relación entre la población de las ciudades capital y la recaudación fiscal, pues el estadístico t fue de -1.113 y su grado de significancia fue de 0.308. Esto confirma lo señalado por Oates (2005) en su crítica a la elección con los pies, de la primera genera- ción teórica de la descentralización, pues el recaudo tributario no parece incidir significativamente en la toma de decisiones a la hora de migrar de una localidad a otra.

En contraste, el análisis de la relación entre población de ciudades principales y gasto público nos permite evidenciar una diferencia sustancial entre América Latina y el resto de los países de la muestra. El grafico 1 presenta el análisis de regresión entre el gasto subnacional y la población de ciudades capitales, introduciendo una variable dummy, denominada país_latinoamerica, que separa los países de América Latina, del resto de la muestra.

El primer elemento que aporta el análisis del gráfico 1 es la concentración de los países de América Latina en la parte superior izquierda, reflejando una menor proporción de gasto público y una mayor concentración demográfica en ciudades capitales, en comparación con los demás países de la muestra. Seguidamente, El gráfico 1 evidencia como los países de América Latina (puntos verdes) tienen un coeficiente de determinación (R2) mucho más bajo que los países por fuera de ese grupo (puntos azules), indicando que el 
modelo de regresión lineal es mucho más explicativo para estos últimos. Esto muestra además cómo, en los países de América Latina, la incidencia del gasto público en la reducción de la primacía urbana de las ciudades principales es mucho menor que la de los demás países.

Grafico 1. Modelo de regresión entre población de ciudades capitales y gasto público descentralizado.

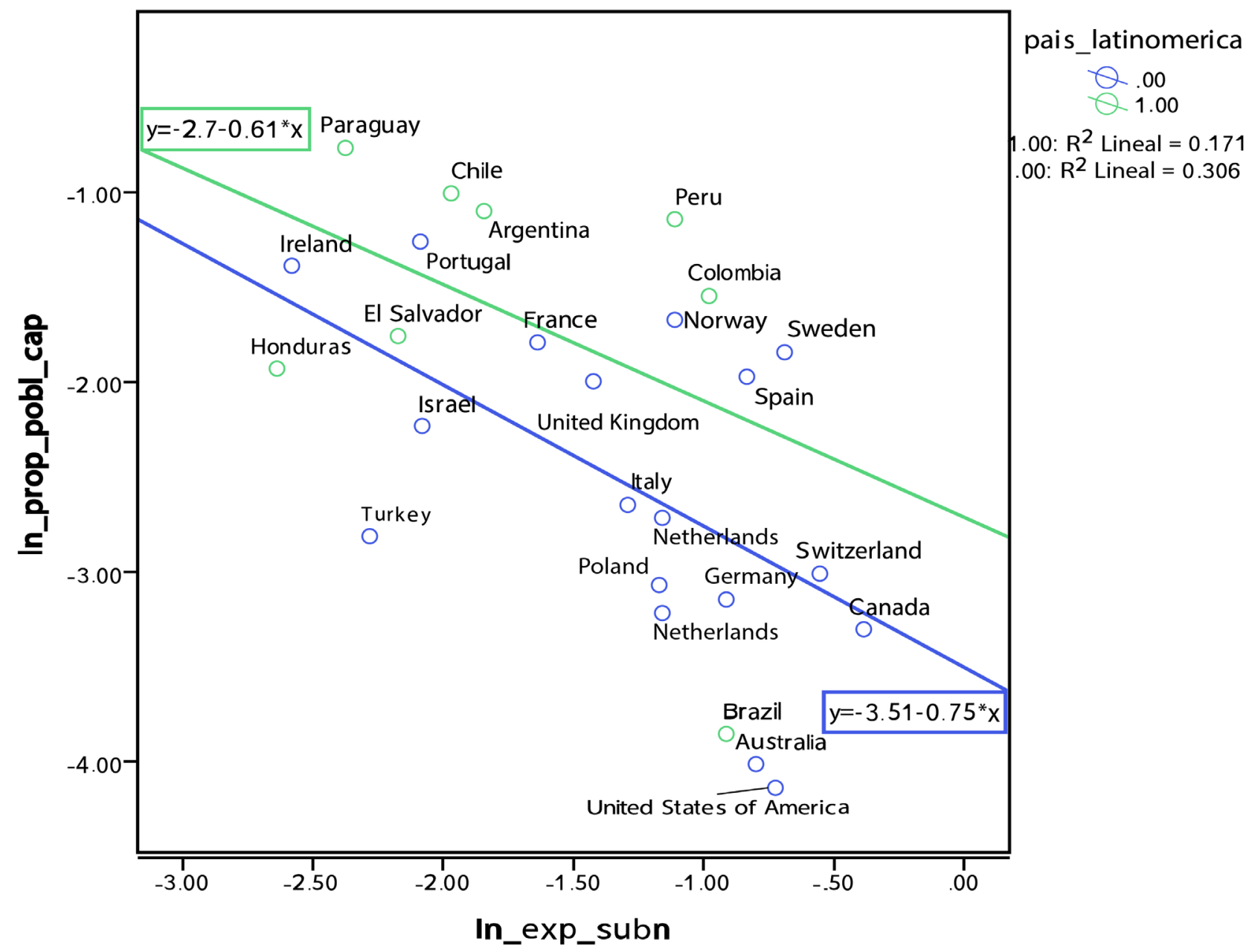

Fuente: Elaboración propia a partir de los datos de IFM, 2018; OCDE, 2016; UN, 2015 
El análisis de regresión revela que la relación entre gasto público descentralizado y la población de la ciudad principal no es explicativa. El estadístico t fue de -1.113 y su grado de significancia fue de 0.308 , con lo cual se descarta una relación estadísticamente significativa entre las dos variables para el grupo de América Latina.

En contraste, los países que no son de América Latina sí presentaron una relación estadísticamente significativa, el estadístico $t$ fue de -2.658 y su grado de significancia fue de 0.017 con lo cual, para estos países, el gasto público descentralizado si tiene un efecto relevante en la reducción de la población de la ciudad principal.

Finalmente es necesario señalar que Brasil es un caso atípico en comparación con el resto de los países de América Latina. El gráfico 1 evidencia que es el único país latinoamericano que presenta una baja proporción de población en su ciudad capital (según el IPT) y su gasto descentralizado es uno de los más altos de la muestra.

Esto se explica entre otras cosas, porque su Distrito Federal (dentro del que se encuentra su capital federal, Brasilia) no es el más poblado, rompiendo la lógica de la primacía urbana. Por el contrario, municipalidades como Sao Pablo, o Rio de Janeiro representan el $5,8 \%$ y el $3,2 \%$ del total de la población, mientras que la capital solo participa con el 1,3\%. Adicionalmente, la importancia histórica y económica de regiones diferentes a la actual capital de Brasil, aunado a la evolución del modelo federal de Brasil y la importancia tributaria de los estados y municipios explica el alto gasto descentralizado del país (IBGE, 2019; Rolnik, 2004).

\section{CONSIDERACIONES FINALES}

La teoría económica indica que la descentralización es un camino para aumentar la eficiencia económica del gasto público y reducir las brechas horizontales en la calidad de vida de los habitantes de las diferentes regiones de cada país. La autonomía local es una alternativa que los gobiernos del mundo han explorado e implementado paulatinamente como una respuesta política a las demandas locales de autonomía, buscando una oferta pública de bienes y servicios más ajustada y pertinente a las preferencias y demandas de la población.

La primera generación teórica de la descentralización fiscal es conocida comúnmente como federalismo fiscal y está basada en los postulados de Tiebout (1959), quien formuló su propuesta argumentando que, si se permite un gasto público descentralizado financiado por impuestos locales, esto respondería más apropiadamente a las preferencias de la población, aumentando la eficiencia del gasto y reduciendo el costo marginal de la tributación, permitiéndole a la ciudadanía movilizarse para residir en la localidad que ofrezca una combinación recaudación/gasto más ajustada a sus preferencias.

Este planteamiento presupone una relación entre concentración demográfica y tributación, señalando a los tributos como un incentivo poderoso para que la población migre de una localidad a otra. De ser real esta hipótesis, la tributación sería (además del mecanismo de financiación del estado), una herramienta de política pública para contrarrestar los efectos de la macrocefalia, conduciendo a las ciudades a tener un tamaño adecuado. 
Sin embargo, este planteamiento se descarta por dos razones.

La primera, porque la teoría de la primacía urbana descarta las presuposiciones normativas para formular la existencia de reglas homogéneas que permitan determinar el tamaño apropiado de una ciudad, por el contrario, reconocen la importancia de analizar de manera contextual y detalla la realidad de cada país, pues el contexto históri$\mathrm{co}$, cultural y geográfico son determinantes para entender las redes urbanas de cada sociedad, y el consiguiente tamaño "optimo" de una ciudad.

Una segunda razón es la inconsistencia de los datos con estas premisas. Al analizar las posibles relaciones estadísticas entre tributación y primacía urbana se encontró que, ni en Latinoamérica ni en el resto de los países de la muestra, la tributación descentralizada coadyuvo a una reducción del dominio de la ciudad principal, por el contrario, la dispersión de los datos conllevó a concluir que la descentralización de la tributación es altamente dispersa entre los países de la muestra y seria espurio señalar una relación estadística con los datos recolectados. Esto aporta al descarte de la hipótesis de Tiebout y reforzaría el razonamiento de Oates, pues los incentivos tributarios no son suficientes para motivar una reconfiguración demográfica, ya que razones culturales, económicas, sociales y geográficas condicionarían esa movilidad interregional.

El teorema de Oates (1999) no desconoce la importancia de la descentralización fiscal, por el contario reafirma la importancia de esta, pero cambia su fundamentación, poniendo énfasis en la redistribución de la ri- queza interregional y no solo en la eficiencia del gasto público.

Oates indica que no es necesaria una reconfiguración demográfica basada en incentivos tributarios, pues en las regiones por fuera de la ciudad principal ya existe una demanda local que, atendida adecuadamente, puede incrementar sus incentivos para no migrara hacia la ciudad capital. El teorema de la descentralización plantea que es Pareto eficiente descentralizar cierto tipo de gasto público en tanto atenderá mejor las preferencias y demandas locales y, por lo tanto, con el mismo nivel de gasto, se aumentará la eficiencia en la atención a la población. Adicionalmente, este tipo de medidas, implementadas en un marco de redistribución de recursos públicos mediante sistemas de transferencias, genera externalidades positivas asociadas a la reducción de brechas horizontales, nuevamente, generando incentivos para evitar la movilidad interregional.

Esta idea cambia el razonamiento y dirige la atención al gasto público, indicando una posible incidencia negativa con la primacía urbana. A mayor gasto de gobiernos subnacionales, menos incentivos para migrar y por consiguiente menor importancia relativa de la ciudad capital, en últimas menos posibilidades de macrocefalia. Al contrastar esta hipótesis con los datos disponibles, se evidencia que es parcialmente cierta.

El análisis de correlaciones indicó alguna incidencia estadística entre el Índice de Población Total (primacía urbana) y el gasto público descentralizado. Sin embargo, al realizar los análisis de regresión por grupos, separando a los países de América Latina del resto, se encontró que solo fue estadística- 


\section{Manuel Alberto Gómez González}

mente relevante para los países no latinoamericanos, pues se encontró una relación estadísticamente significativa, indicando que un aumento en el gasto público ejecutado por los gobiernos locales, si incidió en la reducción de la primacía urbana de los diferentes países no latinoamericanos de la muestra.

Sin embargo, para el caso de los países de América Latina, los datos mostraron un comportamiento diferente. $\mathrm{Ni}$ el análisis de correlación, ni el coeficiente de determinación, ni el estadístico t evidenciaron algún tipo de relación estadísticamente significativa entre el gasto público descentralizado y la reducción de la primacía urbana en América Latina, lo cual puede obedecer a dos razones. Primero, con excepción de Brasil, los países latinoamericanos reflejaron una alta primacía urbana, calificada por autores como macrocefalia, concentrando los países de la región en la parte alta del grafico 1 y confirmando que una de las características sobresalientes de la demografía urbana de América Latina, es la alta concentración en la ciudad principal.

La segunda razón está asociada al gasto público descentralizado, pues, en contraste con el punto anterior, la mayoría de los países (con excepción de Brasil) reflejaron un gasto público descentralizado mucho menor al de los demás países de la muestra. Un bajo gasto público descentralizado y macrocefalia, da como resultado una disminución de la incidencia del primero en la reducción de la segunda.

La bibliografía y el análisis de los datos permiten descartar la incidencia de los tributos locales sobre la reducción de la primacía urbana y la macrocefalia, lo cual rechaza la hipótesis de la primera generación teórica de la descentralización. Sin embargo, la evidencia sí siguiere que el gasto público es una herramienta que la política pública puede usar para influir en la configuración demográfica de las ciudades y la reducción de la primacía urbana. América Latina tiene particularidades que han limitado este efecto, lo cual conlleva a la necesidad de construir un marco teórico y empírico que permita comprender cómo y porqué este efecto difiriere en la región.

Comprender esta relación permitirá a gobiernos y academia incluir al gasto público descentralizado como una herramienta para influir sobre la concentración urbana y la reducción de la macrocefalia. Esta nueva agenda de investigación demanda análisis particulares en cada país y sus regiones, aportando a la formulación de postulados que expliquen y orienten las reformas que los países de la región requieren, buscando potencializar las externalidades positivas de la descentralización fiscal y la reducción de la macrocefalia, aumentando la eficiencia del gasto público y avanzando hacían una mejor calidad de vida en cada localidad, objetivo principal de todos los postulados teóricos analizados.

\section{REFERENCIAS}

Albert, R., Biazzi, R., \& Cabrillo, F. (2011). El Comportamiento de los Gobiernos Autonómicos: Luces y sombras de la descentralización regional. Revista del Instituto de Estudios Económicos, No. 2, pp 213-235. Recuperado de https://dialnet.unirioja.es/ servlet/articulo? codigo $=3759726$

Arango, J. (2003). La Explicación Teórica De Las Migraciones: Luz Y Sombra. Migración 
y Desarrollo, número 001 Red Internacional de Migración y Desarrollo. Recuperado de http://pendientedemigracion.ucm.es/info/ gemi/descargas/articulos/42ARANGO_La_ Explicacion_Teorica_Migraciones_Luces_ Sombras.pdf, DOI: 10.35533/myd.0101.ja

Arrow, K. J. (1970). Political and economic evaluation of social effects and externalities. En The Analysis of Public Output. DOI: https://doi.org/0-87014-220-8.

Braña, F. (2006). Descentralización y eficiencia: Los límites del federalismo económico. Mediterráneo económico, 10, pp. 69-108.

Boisier, S. (1992). ¿Puede la Descentralización Ayudar a la Equidad? Estudios Regionales, No. 32, pp 43-72. Recuperado de http://www.revistaestudiosregionales.com/ documentos/articulos/pdf368.pdf

Castells, M. (1973). Imperialismo y urbanización en América Latina, Editorial Gustavo Gili, Barcelona.

Cibils, V. F., \& Ter-minassian, T. (Editores) (2016). Descentralizando los ingresos fiscales en América Latina: Por qué y cómo. Banco Interamericano de Desarrollo. Recuperado de https://publications.iadb.org/ bitstream/handle/11319/6829/Descentralizando_ingresos_fiscales_America_Latina_Porque_como_Resumen_ejecutivo. pdf? sequence $=4 \&$ isAllowed $=y$

Clemens, J., \& Veldhuis, N. (2013). Federalism and Fiscal Transfers: Essays on Australia, Germany, Switzerland, and United States. Ed.: Fraser Institute, Canadá. Recuperado de https://www.fraserinstitute. org/sites/default/files/federalism-and-fiscal- transfers-rev.pdf

Comité Europeo de las Regiones. (2017). Dictamen del Comité Europeo de las Regiones - Migración en la ruta del Mediterráneo Central. Centre de Documentació Europea,. Recuperado de https://eur-lex.europa. eu/legal-content/ES/TXT/PDF/?uri=CELEX: 52017AR1261\&from $=$ ES

Cortés, A. (2017). Aníbal Quijano: Marginalidad y urbanización dependiente en América Latina. Polis, Revista Latinoamericana, No. 16, pp 221-238. Recuperado de 2019, https://journals.openedition.org/polis/12348. DOI:10.4067/S0718-65682017000100221

Cuervo, L. M. (2004). Desarrollo económico y primac ía urbana en América Latina. Ed.: Red de Bibliotecas Virtuales de CLACSO, Argentina. Recuperado de http://biblioteca.clacso. edu.ar/clacso/gt/20100930123442/5p2art1. pdf

Dughters, R, \& Harper, L. (2004). Descentralización: Un largo processo. Curso La Realidad Fiscal, Módulo 4. Banco Interamericano de Desarrollo (BID) Recuperado de https:// publications.iadb.org/publications/spanish/ document/La-Realidad-Fiscal-Una-Introducción-a-los-Problemas-y-Políticas-Fiscalesen-América-Latina-Módulo-4-Descentralización-Un-largo-proceso.pdf

Finot, I. (2001). Descentralización en América Latina: Teoría y práctica. Comisión Económica para América Latina (CEPAL). Recuperado de https://repositorio.cepal.org/ handle/11362/7261

Flores, M.D. (2002). Federalismo fiscal y asignación de competencias: Una perspecti- 
va teórica. Economía, Sociedad y Territorio, 3. (11). pp 387-407. Recuperado de https:// est.cmq.edu.mx/index.php/est/article/ view/345. DOI: 10.22136/est002002345

Furlan, J. L. (2012). Reforma del Estado, Descentralización Y Gobernabilidad Local en Iberoamérica. Ed: Centro Latinoamericano de Estudios Locales, Argentina. Recuperado de http://www.celadel.org/textos/ estudios1.pdf

Garzarelli, G. (2004). Old and New Theories of Fiscal Federalism, Organizational Design Problems, and Tiebout. Journal of Public Finance and Public Choice, 22 (1-2) Recuperado de https://econwpa.ub.uni-muenchen.de/econ-wp/pe/papers/0509/0509009. pdf. DOI: https://doi.org/10.1332/25156920 4X15668904587133

IBGE - Instituto Brasileiro de Geografia e Estatística (2019). Estimativas Da População Residente Para Os Municípios E Para As Unidades Da Federação Brasileiros Com Data De Referência Em $1^{\circ} \mathrm{De}$ Julho De 2019. Recuperado de https://biblioteca. ibge.gov.br/visualizacao/livros/liv101662.pdf

IFM. Fondo Monetario Internacional (2018). Fiscal Decentralization: IMF's New Global Database. Recuperado de http://data.imf. org/?sk=1C28EBFB-62B3-4B0C-AED3048EEEBB684F

Kahkonen, Satu, \& Lanyi, A. (2001). Descentralización y gestión de gobierno: ¿Contribuye la descentralización a mejorar la prestación de servicios públicos? Banco Mundial. Recuperado de http:// documentos.bancomundial.org/curated/ es/603971468311071050/Descentraliza-
cion-y-gestion-de-gobierno-Contribuye-ladescentralizacion-a-mejorar-la-prestacionde-servicios-publicos

Montoya, J. W. (2009). Globalización, dependencia y urbanización: la transformación reciente de la red de ciudades de América Latina. Revista de geografía Norte Grande, No. 44, pp 5-27. Recuperado de https://scielo. conicyt.cl/scielo.php?script=sci_arttext\&pid $=$ S0718-34022009000300001. DOI: $10.4067 /$ S0718-34022009000300001

Musgrave, R. A., Musgrave, P.B. . (1984). Public Finance in Theory and Practice. Ed.: McGraw-Hil, Estados Unidos. DOI: $10.2307 / 134487$

Niskanen Jr., W. A. (1974). Bureaucracy and Representative Government. The American Political Science Review, 68(4), 1775-1777. DOI: 10.2307/1959997

Oates, W. E. (1974). Fiscal Federalism. The American Political Science Review, 68(4), 1777-1778. DOI: 10.2307/1959998

Oates, W. E. (1999). An Essay on Fiscal Federalism. Jornal of Economic Literature, 37. (3). Pp 1120-1149. Recuperado de http://links. jstor.org/sici?sici=0022-0515\%28199909\%2937\%3A3 \%3C1120\%3AAEOFF\%3E2.0.CO\%3B2-A. DOI: 10.1257/jel.37.3.1120

Oates, W. E. (2005). Toward a second-generation theory of fiscal federalism. International Tax and Public Finance, 12(4), 349-373. DOI https://doi.org/10.1007/s10797-005-1619-9

OCDE. Organización para la Cooperación y el Desarrollo Económicos (2016). Recuperado de http://www.oecd.org/tax/federalism/ 
fiscal-decentralisation-database.htm

OCDE. Organización para la Cooperación y el Desarrollo Económicos (2020). Decentralisation and Regionalisation in Portugal. Recuperado de https://www.oecd-ilibrary. org/urban-rural-and-regional-development/ decentralisation-and-regionalisation-in-portugal_fea62108-en

Qian, Y., \& Weingast, B. R. (1997). Federalism as a Commitment to Preserving Market Incentives. Journal of Economic Perspectives. Recuperado de website: http://pubs. aeaweb.org/doi/10.1257/jep.11.4.83. DOI: 10.1257/jep.11.4.83

Rolnik, R. (2004). Descentralización y Federalismo en el Brasil. Quórum: revista de pensamiento ibero-americano, No 8-9, pp 91-99 Recuperado de https://dialnet.unirioja.es/ descarga/articulo/935612.pdf

Samuelson, P., A. (1955). Diagrammatic exposition of a theory of public expenditure. The Review of Economics and Statistics, 37(4), 350-356. DOI: https://doi. org/10.2307/1925849
Tiebout, C. M. (1959). A Pure Theory of Local Expenditures. The Journal of Political Economy, 64(5), 416-424.DOI: https://doi. org/10.1086/257839

Tsai, H. (1990). Population decentralization policies: The experience of Taiwan. $\mathrm{Zi}$ you Zhongguo zhi, 73(3) pp 1-12. Recuperado de http://europepmc.org/abstract/ MED/12316768

United Nations (2015). World Urbanization Prospects - The 2018 Revision. Department of Economic and Social Affairs Population Division. Recuperado de https://population. un.org/wup/Publications/Files/WUP2018Report.pdf.

Weingast, B. R. (2007). Second Generation Fiscal Federalism: Implications for Decentralized Democratic Governance and Economic Development (2006). Social Science Research Network. Recuperado de https://papers.ssrn.com/sol3/Delivery.cfm/SSRN_ID1153440_code629430. pdf?abstractid $=1153440 \&$ mirid $=1$. DOI: 10.2139/ssrn. 1153440 